\title{
A NOVEL BIO-MICROELECTROMECHANICAL SYSTEM FOR IN VIVO DIAGNOSTIC MONITORING OF FACTURE HEALING
}

\author{
Kirk McGilvray \\ Mechanical Engineering Dept. \\ Colorado State University \\ Fort Collins, CO, USA \\ Emre Unal \\ Electrical and Electronics \\ Engineering Dept. \\ Bilkent University \\ Ankara, Turkey
}

\author{
Hilmi Volkan Demir \\ Electrical and Electronics Engineering Dept. \\ Bilkent University \\ Ankara, Turkey \\ Christian Puttlitz \\ Mechanical Engineering Dept. \\ Colorado State University \\ Fort Collins, CO, USA
}

\section{INTRODUCTION}

The ability to detect a normal from aberrant bone healing cascade during the acute fracture fixation period (1-3 weeks post trauma) is critically important to insure a favorable clinical outcome for many complex fracture cases. Radiographic evaluations do not have the inherent fidelity to make qualitative predictions during the acute healing phase. Early detection of an atypical healing profile would allow for revision strategies to be employed without the need for expensive and undesirable followup surgeries. In an effort to address the critical need to diagnose the course of bone fracture healing in the vitally important early healing phase, our research group has developed a radio frequency (RF) strain sensor that takes advantages of the recent advances in meta-materials and micro-electo-mechanical systems (MEMS) technology. Our MEMS sensor is biocompatible (bioMEMS), inductively powered and monitors the surface strains on implanted hardware $[1,2]$. Another novel feature of this new sensor is that it does not require an internal-external physical connection to sense and transmit in vivo biological data. The essence of the sensor's design is that straining the integrated RF bioMEMS circuit results in a shift in its resonant frequency (ResF). Through telemetric detection of this ResF shift, it is possible to longitudinally monitor the temporal changes in hardware strain. It is well understood that that as proper fracture healing progresses (i.e. increasingly stable tissue(s) stabilizing the fracture sight) that the load/strain born by orthopaedic implant diminishes. Therefore, telemetric measurements of our bioMEMS system (i.e. hardware load/strain) provide direct insight into the degree of fracture stabilization and healing.

\section{METHODS}

A standard locking fracture fixation plate (Synthes, West Chester, PA) with a bioMEMS sensor attached to the plate's surface was used to stabilize an osteotomy in an IACUC-approved ovine metatarsal model. Two treatment groups were evaluated: (1) a non-critical defect $(4.8 \pm 1.1 \mathrm{~mm} ; \mathrm{n}=7)$ and (2) a critical sized defect $(13.8 \pm 1.0 \mathrm{~mm} ; \mathrm{n}=7)$ wherein it was expected that calcified tissue would and would not bridge the osteotomy, respectively, after six weeks of convalescence (Figure 1). Bi-weekly ResF readings were recorded from the implanted sensor from the surgery data until euthanasia using a custom-built, near-field antenna and network analyzer/computer interface. The operated hind limb was cyclically loaded $(n=10)$ at $100 \mathrm{~N}$ increments from $0-400 \mathrm{~N}$ using a manually driven worm-drive platform with attached force sensor. The ratio between the applied load and the shift in the ResF under loading of the bioMEMS sensor was calculated for the last five cycles and averaged (daily ResF magnitude shift, DRFM). A decrease in the DRFM under the same applied load over time indicated a reduction with respect to the in vivo hardware stain, and represented a measure of fracture stabilization and healing. The data were normalized to the maximum DRFM value for each animal, and the resultant linear representation of the normalized DRFM (nDRFM) was used to compare the sensor differential response between the two treatment groups. A Student-NewmanKeuls statistical test was performed post-hoc to determine significant differences between the two groups (threshold was set at $\mathrm{p}$-value $<0.05$ ), where nDRFM was the variable of interest. Post sacrifice micro-computed tomography and histomorphometry parameters were used to correlate the sensor's response to tissue mineral and fibrous composition.

\section{RESULTS}

The data demonstrated a gradual reduction of the nDRFM [(ResF/applied load)/(days post-surgery)] response as fracture healing progressed for both groups (Figure 3). The nDRFM data during the first month of healing indicated a statistically significant difference ( $\mathrm{p}$-value $=0.02 ; 44 \%$ ) between the non-critical ($0.034 \pm 0.017 \quad[(\mathrm{MHz} / \mathrm{N}) / \mathrm{Day}])$ and critical $(-0.019 \pm 0.003$ 
$[(\mathrm{MHz} / \mathrm{N}) / \mathrm{Day}]$ ) treatments (Figure 3). Micro-computed tomography data demonstrated complete bridging of the osteotomy with ossified tissue for all of the non-critically sized osteotomies and none of the critically-sized fractures demonstrated bridging of the fracture gap indicating the models accurately represented the normal and aberrant healing variants (Figure 2). The nDRFM response of the sensor at sacrifice ( $45 \pm 8$ days post-surgery) was plotted against the histomorphic data (Figure 3) and the data provided a high correlation between the bioMEMS sensor response and percent bone at the fracture site $\left(r^{2}>0.91\right)$ These data directly demonstrate the sensor's fidelity to detect the biomechanical stability at the fracture site following extended healing.

\section{DISCUSSION}

Using a fixation plate with a wired strain gage, Stoffel et al. [3] demonstrated similar patterns of decreasing plate strain magnitude during the healing cascade in an ovine tibia model. Our data demonstrated the feasibility of using a wireless bioMEMS sensor to predict and measure both the acute and chronic biomechanical changes observed during healing cascade of long bone fracture.

\section{REFERENCES}

[1.].Melik, R., et al., Metamaterial based telemetric strain sensing in different materials. Optics Express, 2010. 18(5): p. 5000-5007. [2.] Melik, R., et al., Nested Metamaterials for Wireless Strain Sensing. IEEE Journal of Selected Topics in Quantum Electronics, 2010. 16(2): p. 450-458. [3.]Stoffel, K., K. Klaue, and S.M. Perren, Functional load of plates in fracture fixation in vivo and its correlate in bone healing. Injury, 2000. 31 Suppl 2: p. S-B37-50.

\section{ACKNOWLEDGEMENTS}

Financial support provided by the National Institutes of Health (EB0121048).

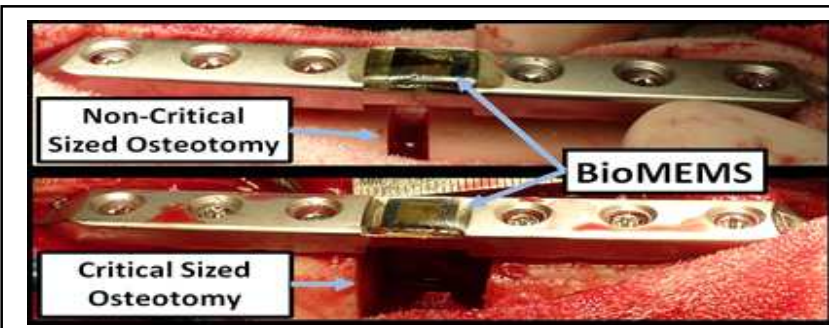

Figure 1: BioMEMS adhered to six-hole locking plate. Both critically sized and non-critical osteotomy models can be seen.

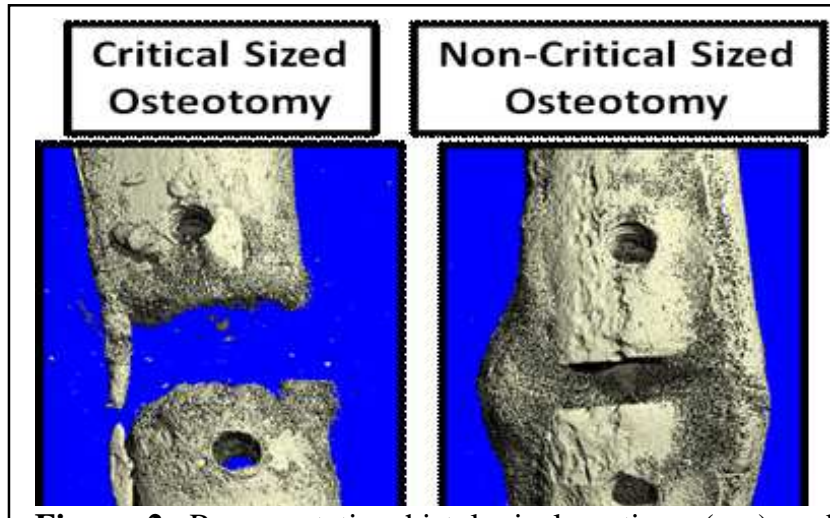

Figure 2: Representative histological sections (top) and 3D micro-computed tomography reconstructions (bottom) demonstrating the differences between a critically sized and non-critically sized defect at sacrifice.

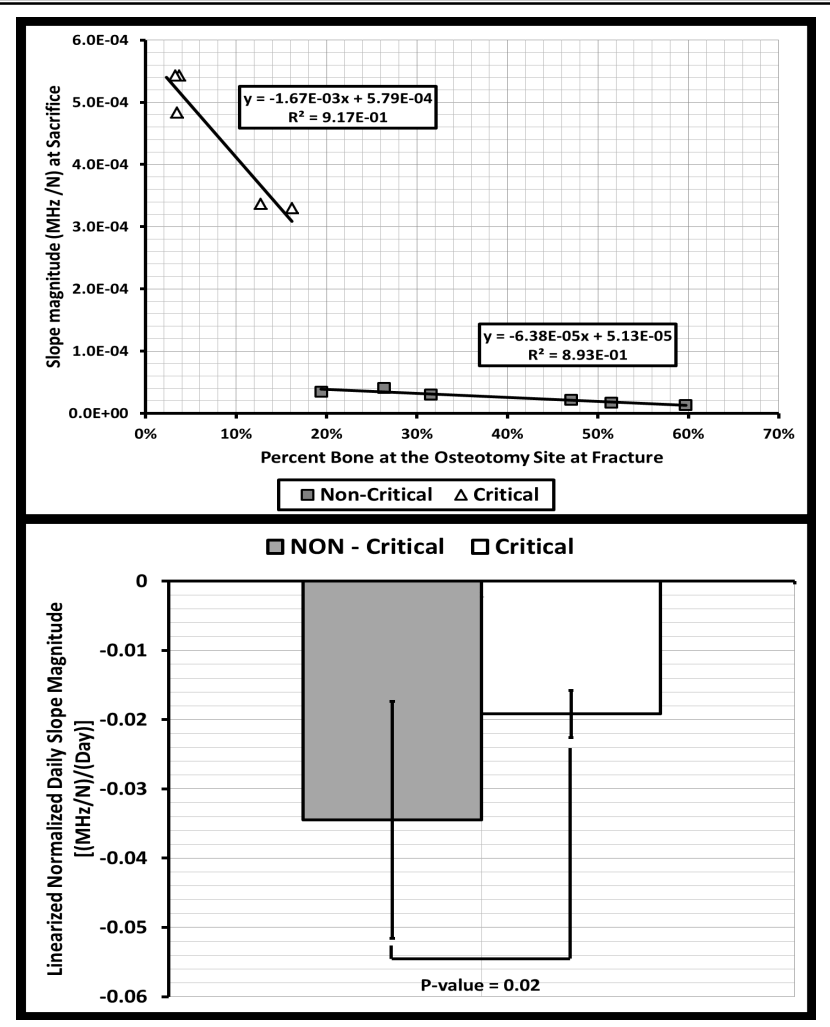

Figure 3: (TOP). Histomorphometric data showed that the critical sized osteotomy had statistically less bone at the fracture site $(81 \%$ decrease) compared to the non-critical sized treatment ( $p$-value $<0.001)$. The nDRFM response of the sensor at sacrifice was plotted against the histomorphometric data and was used to correlate the sensor's response to the osteotomies composition. (BOTTOM) nDRFM data means shown with standard deviation bars for both model types, demonstrating significant differences between treatments during the acute (initial 30 days post surgery) (p-value $=0.02$ ). 